\title{
Volatility of Volatility of Financial Markets
}

\author{
L. INGBER \\ DRW Investments LLC, Chicago Mercantile Exchange Center \\ 30 S. Wacker Dr., Ste 1516, Chicago, IL 60606, U.S.A. \\ and \\ Lester Ingber Research \\ P.O. Box 06440, Wacker Dr. PO - Sears Tower, Chicago, IL 60606-0440, U.S.A. \\ ingber@ingber.com ingber@alumni.caltech.edu \\ J. K. WILSON \\ DRW Investments LLC, Chicago Mercantile Exchange Center \\ 30 S. Wacker Dr., Ste. 1516, Chicago, IL 60606, U.S.A. \\ jenwilson1@yahoo.com
}

(Received and accepted April 1998)

\begin{abstract}
We present empirical evidence for considering volatility of Eurodollar futures as a stochastic process, requiring a generalization of the standard Black-Scholes (BS) model which treats volatility as a constant. We use a previous development of a statistical mechanics of financial markets (SMFM) to model these issues. (C) 1999 Elsevier Science Ltd. All rights reserved.
\end{abstract}

Keywords-Options, Eurodollar, Volatility, Statistical mechanics.

\section{INTRODUCTION}

There always is much interest in developing more sophisticated pricing models for financial instruments. In particular, there currently is much interest in improving options pricing models, particularly with respect to stochastic variables [1-3].

In Section 2, we outline the nature of options pricing on a particular set of futures contracts, Eurodollars. In Section 3, as an introduction to the mathematics of options pricing, we outline the Black-Scholes (BS) model of options. In Section 4, we describe some generalizations to the BS model, including time-dependent volatility, and we introduce the path-integral representation of BS-type equations, useful for our present development.

In Section 5, we give graphical results of a scries of straightforward statistical analyses of Eurodollar contracts, leading to the conclusion that indeed volatility is quite likely a stochastic process. In Section 6, we describe how stochastic volatility, e.g., "volatility of volatility", can be modelled within our framework of statistical mechanics of financial markets (SMFM) [4-8]. In Section 7, we present our conclusion.

We thank Donald R. Wilson for his support and discussions on trading Eurodollar options.

We thank Man Wei Tam at DRW Investments for his work in preparing the data and for discussions throughout the course of this project. Yield data was extracted from the MIM database of Logical Information Machines (LIM). Implied volatility data was extracted from the database of Bridge/Knight-Ridder. 
In the course of this work, we were surprised to learn that many people involved in trading as well as in data preparation did not understand the use of the " $n /(n-1)$ " in calculating the "unbiased standard deviation", though this algorithm is used in practically every finance textbook. Since this journal reaches an interdisciplinary audience, in the Appendix a short derivation is given for this unbiased estimator.

\section{OUTLINE OF OPTIONS}

\subsection{Eurodollars}

Eurodollars are fixed-rate time deposits held primarily by ovcrscas banks, but denominated in U.S. dollars. They are not subject to U.S. banking regulations and therefore tend to have a tighter bid-ask spread than deposits held in the United States [9].

\subsection{Futures}

The three-month Eurodollar futures contract is one of the most actively traded futures markets in the world. The contract is quoted as an index where the yield is equal to the Eurodollar price subtracted from 100. This yield is equal to the fixed rate of interest paid by Eurodollar time deposits upon maturity and is expressed as an annualized interest rate based on a 360-day year. The Eurodollar futures are cash settled based on the 90-day London Interbank Offer Rate (LIBOR). A "notional" principal amount of $\$ 1$ million, is used to determine the change in the total interest payable on a hypothetical underlying time deposit, but is never actually paid or received [9].

Currently a total of 40 quarterly Eurodollar futures contracts (or ten years worth) are listed, with expirations quarterly in March, June, September, and December.

\subsection{Options on Futures}

The options traded on the Eurodollar futures include not only 18 months of contracts expiring at the same time as the underlying future, but also various short dated options which themselves expire up to one year prior to the expiration of the underlying futures contract.

\subsection{Front/Back Month Contracts}

For purposes of risk minimization, as discussed below, traders put on spreads across a variety of options contracts. One common example is to trade the spread on contracts expiring one year apart, where the future closer to expiration is referred to as the front month contract, and the future expiring one year later is called the back month. The availability of short dated or "midcurve" options which are based on an underlying back month futures contract, but expire at the same time as the front month, allow one to trade the volatility ratios of the front and back month futures contracts without having to take the time differences in options expiration into consideration. It is the volatilities of these types of front and back month contracts which are used for the study in this paper.

\section{STANDARD BLACK-SCHOLES (BS) MODEL}

The standard partial-differential equation used to formulate most variants of Black-Scholes (BS) models describing the market value of an option, $V$, is

$$
\frac{\partial V}{\partial t}+\frac{1}{2} \sigma^{2} S^{2} \frac{\partial^{2} V}{\partial S^{2}}+r S \frac{\partial V}{\partial S}-r V=0
$$

where $S$ is the asset price, and $\sigma$ is the standard deviation, or volatility of $S$, and $r$ is the short-term interest rate. The solution depends on boundary conditions, subject to a number of 
interpretations, some requiring minor transformations of the basic BS equation or its solution. For example, the basic equation can apply to a number of one-dimensional models of interpretations of prices given to $V$, e.g., puts or calls, and to $S$, e.g., stocks or futures, dividends, etc.

For instance, if $V$ is set to $C$, a call on an European option with exercise price $E$ with maturity at $T$, the solution is

$$
\begin{aligned}
C(S, t) & =S N\left(d_{1}\right)-E e^{-r(T-t)} N\left(d_{2}\right), \\
d_{1} & =\frac{\ln (S / E)+\left(r+(1 / 2) \sigma^{2}\right)(T-t)}{\sigma(T-t)^{1 / 2}}, \\
d_{2} & =\frac{\ln (S / E)+\left(r-(1 / 2) \sigma^{2}\right)(T-t)}{\sigma(T-t)^{1 / 2}} .
\end{aligned}
$$

In practice, the volatility $\sigma$ is the least known parameter in this equation. Estimation of $\sigma$ is most often the most important part of pricing options. Usually the volatility is given in a yearly basis, baselined to some standard such as 252 (or 250 or 260, etc.) trading days per year. Therefore, all values of volatility given in the graphs in this paper, based on daily data, would be annualized by multiplying the standard deviations of the yields by $\sqrt{252}=15.87$. We have used this factor to present our implied volatilities as daily movements.

\subsection{Some Key Issues in Derivation of BS}

The basic BS model considers a portfolio in terms of delta $(\Delta)$,

$$
\Pi=V-\Delta S
$$

in a market with Gaussian-Markovian ("white") noise $X$ and drift $\mu$,

$$
\frac{d S}{S}=\sigma d X+\mu d t
$$

where $V(s, T)$ inherits a random process from $S$,

$$
d V=o S \frac{\partial V}{\partial S} d X+\left(\mu S \frac{\partial V}{\partial S}+\frac{1}{2} \sigma^{2} S^{2} \frac{\partial^{2} V}{\partial S^{2}}+\frac{\partial V}{\partial t}\right) d t
$$

This yields

$$
d \Pi=\sigma\left(\frac{\partial V}{\partial S}-\Delta\right) d X+\left(\mu S \frac{\partial V}{\partial S}+\frac{1}{2} \sigma^{2} S^{2} \frac{\partial^{2} V}{\partial S^{2}}+\frac{\partial V}{\partial t}-\mu \Delta S\right) d t
$$

The expected risk-neutral return of $\Pi$ is

$$
d \Pi=r \Pi d t=r(V-\Delta S) d t .
$$

Options $V$ on futures $F$ can be derived, e.g., using simple transformations such as

$$
F=S e^{r(T-t)}
$$

and setting

$$
d \Pi=r V d t .
$$

The corresponding BS equation for futures $F$ is

$$
\frac{\partial V}{\partial t}+\frac{1}{2} \sigma^{2} F^{2} \frac{\partial^{2} V}{\partial S^{2}}-r V=0
$$


At least two advantages are present if $\Delta$ is chosen such that

$$
\Delta=\frac{\partial V}{\partial S}
$$

Then, the portfolio can be instantaneously "risk-neutral," in terms of zeroing the coefficient of $X$, as well as independent of the direction of market, in terms of zeroing the coefficient of $\mu$. For the above example of $V=C$,

$$
\Delta=N\left(d_{1}\right)
$$

Other trading strategies based on this simple model are based on hedging with other similar constructs, e.g., using gamma $(\Gamma)$, theta $(\Theta)$, vega, rho $(\rho)[10]$,

$$
\begin{aligned}
\Gamma & =\frac{\partial^{2} \Pi}{\partial S^{2}}, \\
\Theta & =\frac{\partial \Pi}{\partial t}, \\
\operatorname{vega} & =\frac{\partial \Pi}{\partial \sigma}, \\
\rho & =\frac{\partial \Pi}{\partial r} .
\end{aligned}
$$

The BS equation, equation (1), may be written as

$$
\Theta+r S \Delta+\frac{1}{2}(\sigma S)^{2} \Gamma=r f
$$

\section{GENERALIZATION OF BLACK-SCHOLES MODEL}

\subsection{Time Dependent $\sigma(t)$ and $r(t)$}

The volatility $\sigma$ may depend on other variables, and the BS model may be generalized to multivariable models. However, within the framework of the basic BS model, if $\sigma$ and $r$ are timedependent, then it turns out that the above solutions of the basic BS, and the use of the above set of $\{\Delta, \Gamma, \Theta$, vega, $\rho\}$, etc., can be used without change, provided an "effective" volatility, $\hat{\sigma}$, is defined in terms of $\sigma(t)$, and an "effective" interest rate, $\hat{r}$, is defined in terms of $r(t)$, are defined.

This can be developed by considering a slight generalization of the above BS equation for the variable $\hat{V}$, using methods given in a standard text [11]. A transformation of variables is used to transform away any time-dependent coefficients,

$$
\begin{gathered}
\hat{S}=S e^{\alpha(t)}, \\
\hat{V}=V e^{\beta(t)}, \\
\hat{t}=\gamma(t) .
\end{gathered}
$$

This leads to

$$
\frac{d \gamma(t)}{d t} \frac{\partial \hat{V}}{\partial \hat{t}}+\frac{1}{2} \sigma(t)^{2} \hat{S}^{2} \frac{\partial^{2} \hat{V}}{\partial \hat{S}^{2}}+\left(r(t)+\frac{d \alpha(t)}{d t}\right) \hat{S} \frac{\partial \hat{V}}{\partial \hat{S}}-\left(r(t)+\frac{d \beta(t)}{d t}\right) \hat{V}=0
$$

Taking

$$
\begin{aligned}
& \alpha(t)=\int_{t}^{T} r(\tau) d \tau, \\
& \beta(t)=\int_{t}^{T} r(\tau) d \tau, \\
& \gamma(t)=\int_{t}^{T} \sigma^{2}(\tau) d \tau,
\end{aligned}
$$


leads to

$$
\frac{\partial \hat{V}}{\partial \hat{t}}=\frac{1}{2} \hat{S}^{2} \frac{\partial^{2} \hat{V}}{\partial \hat{S}^{2}}
$$

with coefficients independent of time $t$ (the motivation for this transformation). In terms of $V$,

$$
V(S, t)=e^{-\beta(t)} \hat{V}\left(S e^{\alpha(t)}, \gamma(t)\right)
$$

To get the explicit form of the solution, consider the original BS model with constant coefficients and solution $V_{B S}$ :

$$
V_{B S}=e^{-(T-t) r} \hat{V}_{B S}\left(S e^{-(T-t) r},(T-t) \sigma^{2}\right),
$$

for some solution $\hat{V}_{B S}$. Therefore, the standard BS solutions for various products can be used if $\sigma$ and/or $r$ are time-dependent, by replacing $\sigma$ in the original equation by $\hat{\sigma}$, and by replacing $r$ in the original equation by $\hat{r}$,

$$
\begin{aligned}
\sigma^{2} \rightarrow \hat{\sigma}^{2} & =\frac{1}{T-t} \int_{t}^{T} \sigma^{2}(\tau) d \tau, \\
r \rightarrow \hat{r} & =\frac{1}{T-t} \int_{t}^{T} r(\tau) d \tau
\end{aligned}
$$

\subsubsection{Example of use}

This can be very useful in conjunction with models of time-dependent volatilities and interest rates. For example, if volatilities are fit to data, and a moving-average optimization gives a form like

$$
\sigma(t)=A(T-t)^{z}
$$

then the effective $\hat{\sigma}$ to use for the day's trading would be the scaled $\sigma(t)$,

$$
\hat{\sigma}=\frac{A(T-t)^{z}}{\sqrt{2 z+1}}=\frac{\sigma(t)}{\sqrt{2 z+1}} .
$$

\subsection{Some Discretization Issues}

Since tree approximations and/or discretization of variables are often applied to calculating the above entities, it is useful to apply limits to some of these discretizations wherever possible [12-14].

Equation (1) has another mathematically equivalent path-integral representation [15], which often offers some advantages in formulation, calculation, and inclusion of boundary conditions. Here, attention is drawn to the derivation of limits on the meshes of $\delta t$ and $\delta v$ such that there can be maintained numerical equivalence between these equivalent algebraic representations.

\subsection{Path-Integral Representation}

The short-time conditional representation of $V$ in an epoch $\delta t$ is given in terms of the "Lagrangian" $L$ in the Itô prepoint discretization,

$$
\begin{aligned}
V[S, t+\delta t \mid S, t] & =\left(2 \pi(\sigma S)^{2} \delta t\right)^{-1 / 2} \exp (-L \delta t) \\
L & =\frac{(\dot{S}+r S)^{2}}{2(\sigma S)^{2}}+r \\
\dot{S} & =\frac{\delta S}{\delta t}=\frac{S(t+\delta t)-S(t)}{\delta t} .
\end{aligned}
$$


For equation (1), the condition on the mesh of $\delta t$ is that it be no coarser than

$$
\delta t \leq \frac{2(\sigma S)^{2}}{(r S)^{2}}=\frac{2 \sigma^{2}}{r^{2}}
$$

throughout the ranges of $S$ giving the most important contributions to $V$, e.g., where $\dot{S}$ is small. The mesh of $S$ is optimally chosen such that $\delta S$ is measured by the variance $(\sigma S)^{2}$,

$$
\delta S \approx \sigma S(\delta t)^{1 / 2}
$$

For equation (10), similarly, the mesh of $F$ is measured by the variance,

$$
\delta F \approx \sigma F(\delta t)^{1 / 2}
$$

However, here there is no interest rate drift term, the above criteria for $\delta t$ is not applicable, and so

$$
\delta t \leq \frac{2(\sigma F)^{2}}{\dot{F}^{2}}
$$

for some "typical" $\dot{F}$.

Note that if $\sigma$ and $r$ are time-dependent, then, as discussed above, $\hat{\sigma}$ and $\hat{r}$ are the appropriate variables to use in these constraints on the meshes. Also, in the region of boundary and final conditions, often tighter meshes are required.

\subsection{Itô Representation Transformations}

Some care must be taken with nonconstant drifts and diffusions. For example, for purposes of calculating volatilities, it is often convenient to transform to a variable $Z$

$$
Z=\ln S
$$

The above distribution can be transformed into $V[Z, t+\delta t \mid Z, t]$,

$$
\begin{aligned}
d S_{t} V[S, t+\delta t \mid S, t] & =d Z_{t} V[Z, t+\delta t \mid Z, t] \\
& =d Z_{t}\left(2 \pi \sigma^{2} \delta t\right)^{-1 / 2} \exp \left(-L^{\prime} \delta t\right) \\
L^{\prime} \delta t & =\frac{\left(\left[\exp \left(Z_{t+\delta t}-Z_{t}\right)-1\right]+r \delta t\right)^{2}}{2 \sigma^{2} \delta t}+r \delta t
\end{aligned}
$$

This can be expanded into

$$
\begin{aligned}
L^{\prime} \delta t & \approx \frac{\left(Z_{t+\delta t}-Z_{t}+(1 / 2)\left(Z_{t+\delta t}-Z_{t}\right)^{2}+r \delta t\right)^{2}}{2 \sigma^{2} \delta t}+r \delta t \\
& \approx \frac{\left(Z_{t+\delta t}-Z_{t}+\left(r+(1 / 2) \sigma^{2}\right) \delta t\right)^{2}}{2 \sigma^{2} \delta t}+r \delta t \\
\left(Z_{t+\delta t}-Z_{t}\right)^{2} & \approx \sigma^{2} \delta t,
\end{aligned}
$$

where only terms of order $\delta t$ have been kept, yielding

$$
L^{\prime}=\frac{\left(\dot{Z}+\left(r+(1 / 2) \sigma^{2}\right)\right)^{2}}{2 \sigma^{2}}+r
$$

This defines the distribution $V[Z, t+\delta t \mid Z, t]$ in terms of the distribution $V[S, t+\delta t \mid S, t]$, where the volatilities differ by a factor of $S^{-1}$ and the drifts differ by the term $(1 / 2) \sigma^{2}$. The 


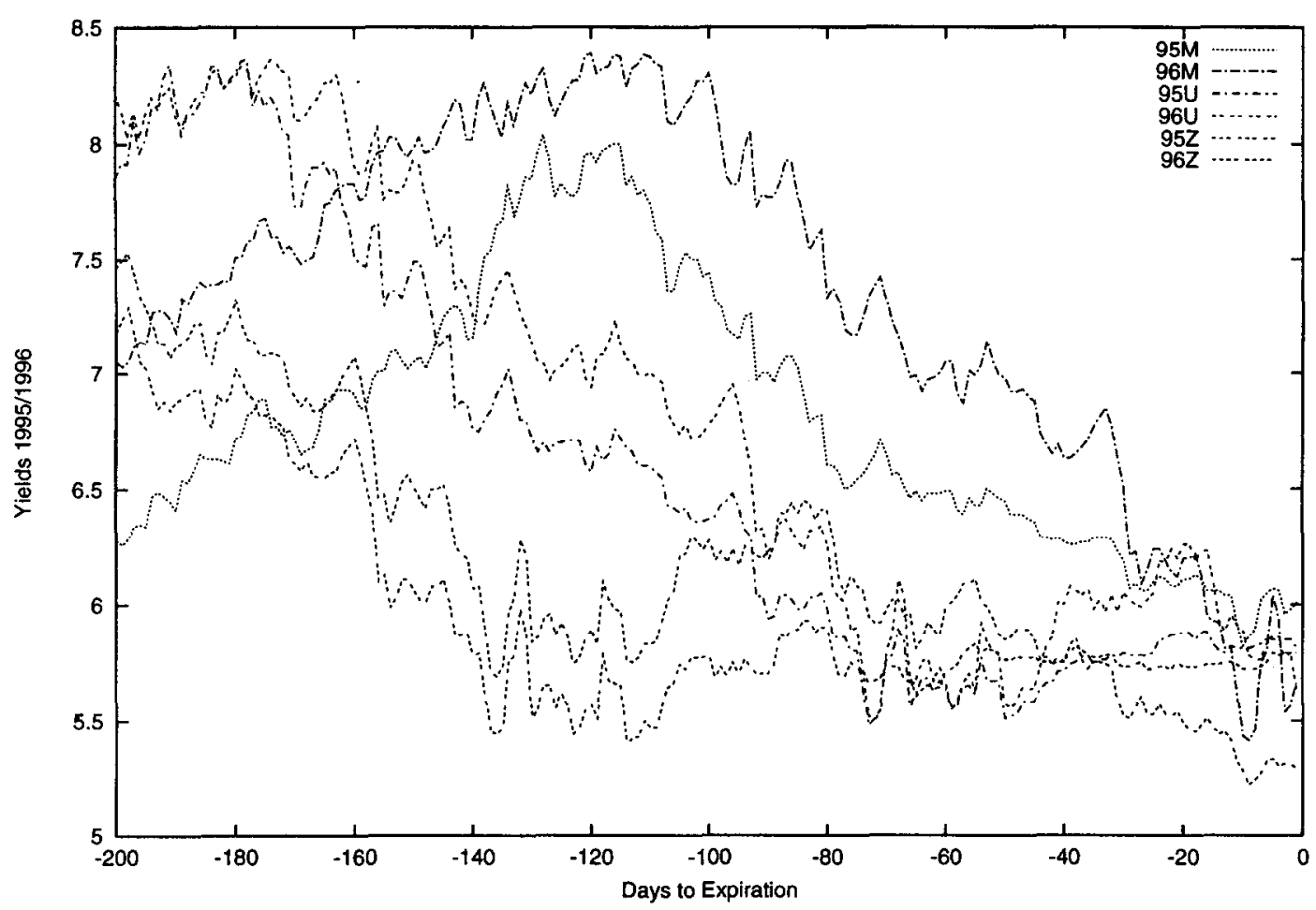

Figure 1. Front and back month 1995 yields, marked to the number of days to expiration of the front contract.

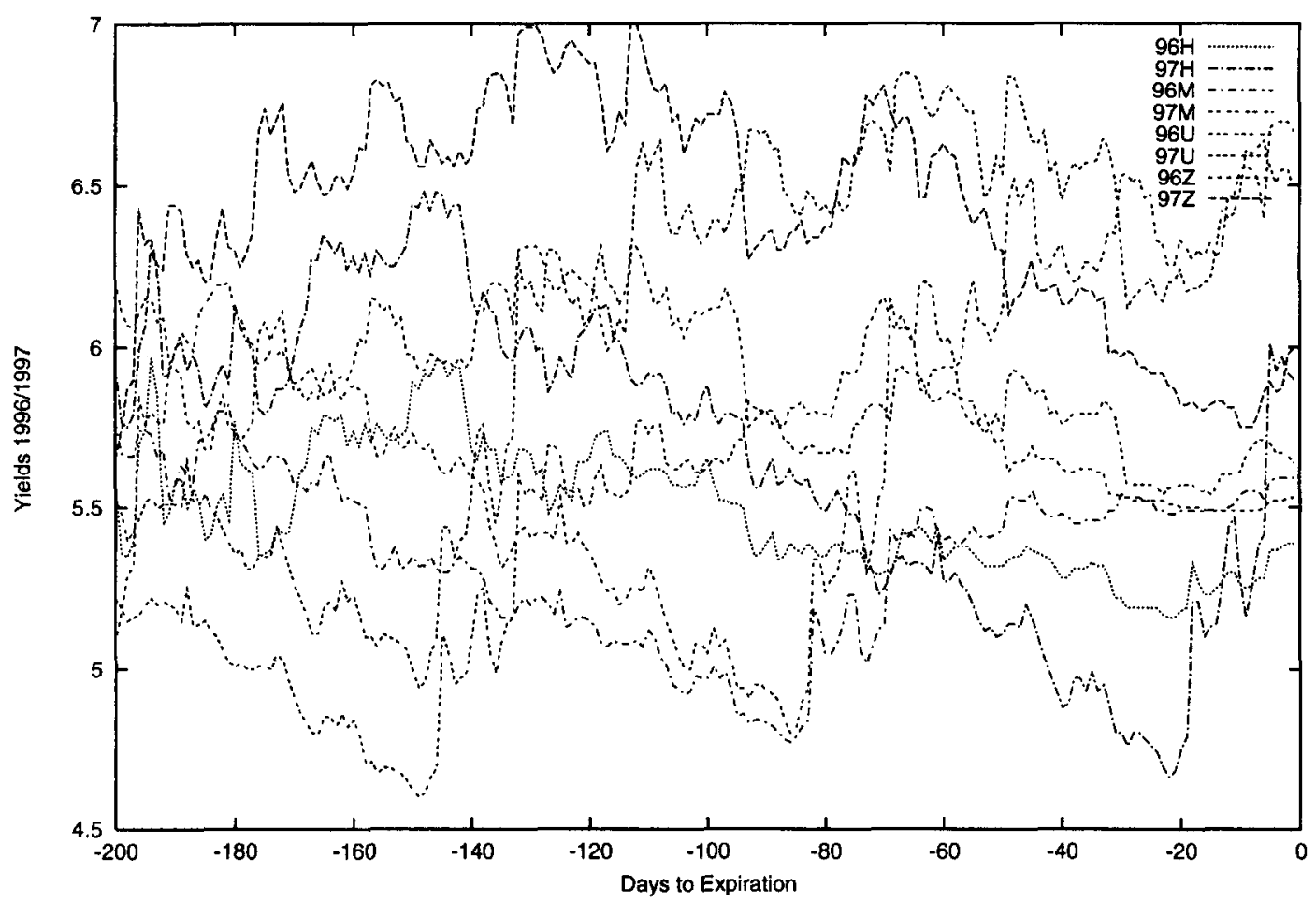

Figure 2. Front and back month 1996 yields, marked to the number of days to expiration of the front contract.

above procedure is valid in the multivariate case for more general nonlinear drifts and diffusions to order $\delta t^{3 / 2}[15]$. 


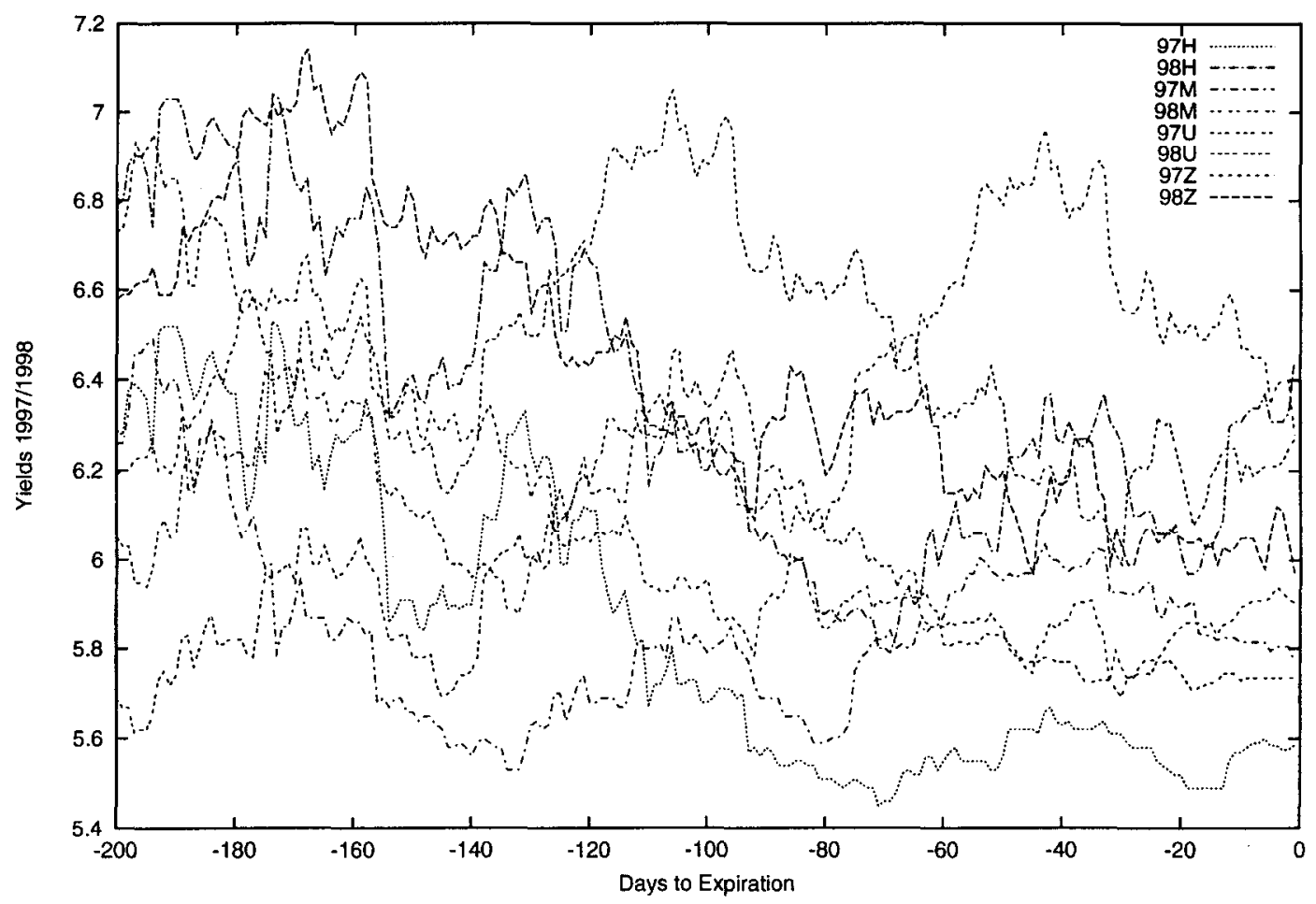

Figure 3. Front and back month 1997 yields, marked to the number of days to expiration of the front contract.

\section{STATISTICS OF EURODOLLAR CONTRACT DATA}

In the following analyses, it should be clear that the correlations we observe are specific to the time scales and the windows used for averaging out data. These scales and windows have been selected because of their widespread use in actual trading. However, this may not imply correlations at other times scales or windows [16].

\subsection{Yields}

Figures 1-3 show the front and back month yields, marked to the number of days to expiration of the front contract, for years 1995, 1996, and 1997, respectively.

\subsection{Volatility}

The historical volatility is relatively insensitive to the window used for taking sample data.

Figure 4 illustrates the window sensitivity in volatility calculations.

\subsubsection{Basis-Point Volatility (BPV) versus Black-Scholes Volatility (BSV)}

The basis-point volatility (BPV) is derived from the observed sample data of daily differenced yields $(d t=1)$, e.g.,

$$
\mathrm{BPV}=\left\langle\frac{n}{n-1}(d y-\langle d y\rangle)^{2}\right\rangle^{1 / 2}
$$

for a sample size of $n$ points of data. In the context of equation (1) above, given that the BPV is calculated over a sample of daily data, e.g., 20 days,

$$
\mathrm{BPV} \approx\langle y\rangle \sigma .
$$

The BPV is a more natural measure of the movement of the yields and is used more by traders than by theorists. We regularly draw comparisons in our calculations between the BPV and 


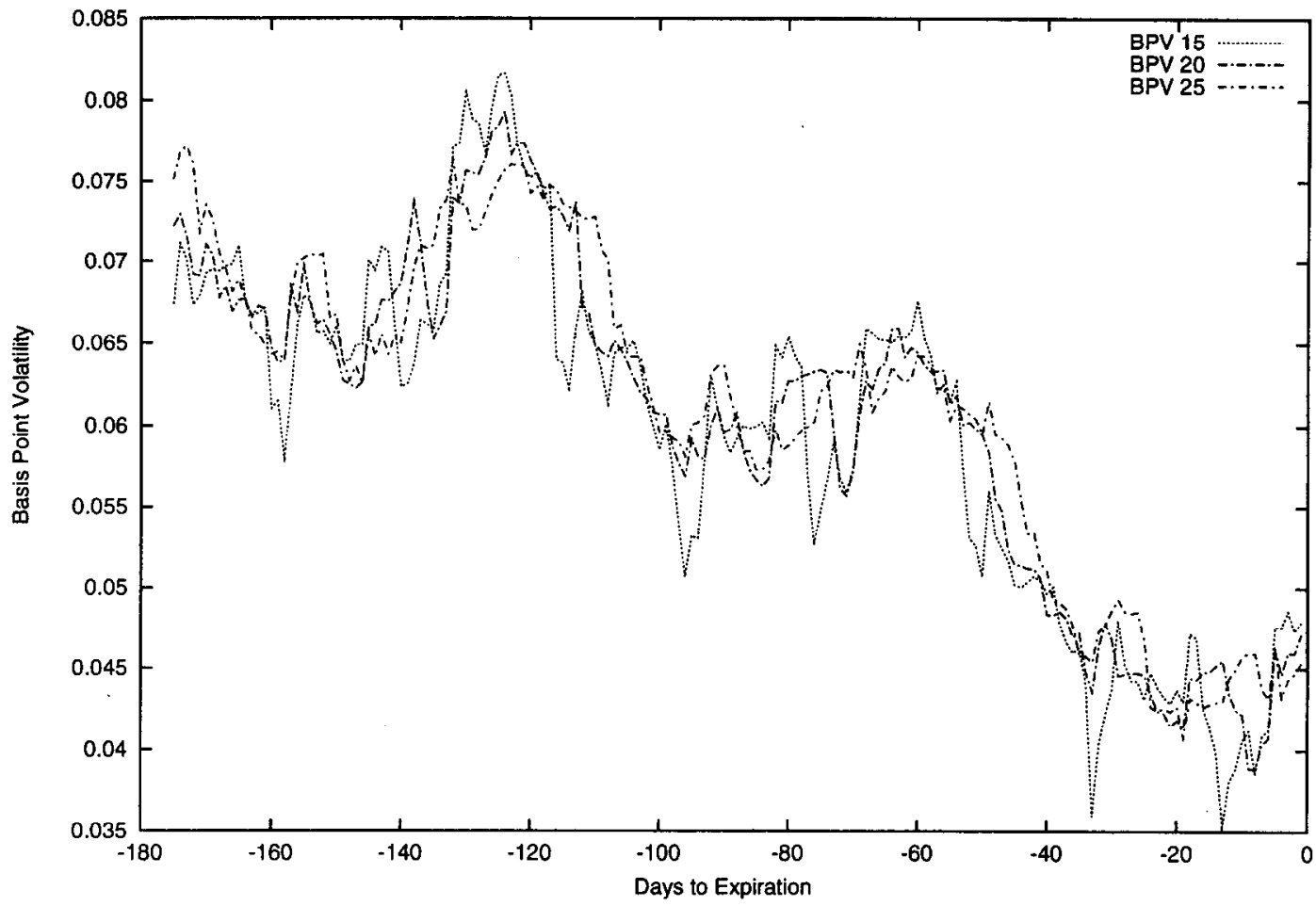

Figure 4. Illustration of window sensitivity in volatility calculations, marked to the number of days to expiration of the front contract.

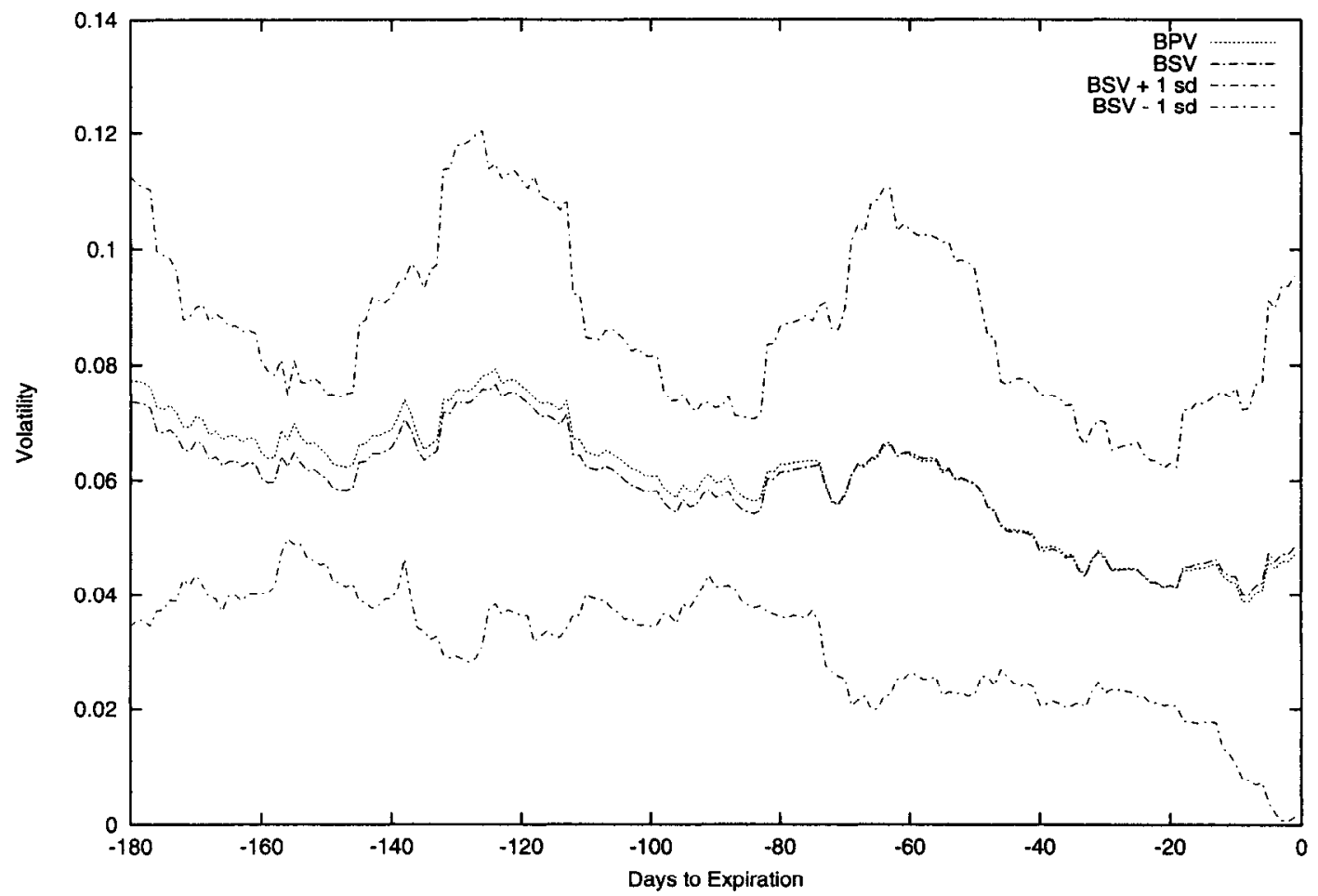

Figure 5. Basis-Point Volatilities (BPV) and Black-Scholes Volatilities (BSV) with bands of the BSV standard deviations (SDBPV) across all contract months, marked to the number of days to expiration of the front contract. The BSV and the SDBPV have been scaled to the BPV and the SDBPV by multiplying them by the rounded average of the yields, i.e., 6.0 . 
the BS volatility, i.e., $\sigma$, to be sure that we are not inducing some effects by the choice of one volatility over the other.

We first take standard deviations of the volatilities of each contract, then take the average over similar contracts, e.g., with the same number of days until expiration. This establishes that there is a distribution of volatilities over similar contracts, beyond the act of their aggregation.

Figure 5 shows Basis-Point Volatilities (BPV) and Black-Scholes Volatilities (BSV) with bands of the BSV standard deviations (SDBSV) across all contract months. (The BSV bands are smaller than the BPV bands.) The BSV and the SDBSV have been scaled to the BPV and the SDBPV by multiplying them by the rounded average of the yields, i.e., 6.0. Note that after scaling, they consistently lie within their standard deviations.

\subsubsection{Volatility ratios}

Trading on the volatility ratios of front/back contracts often presents less risk than trading on the separate contracts. Let $w(x, y)$ be a function of two random variables (say, $w=x / y$, like front and back contracts). Then,

$$
\begin{aligned}
w & =\langle w\rangle+(x-\langle x\rangle) \frac{\partial w}{\partial x}+(y-\langle y\rangle) \frac{\partial w}{\partial y}+\ldots \\
\langle w\rangle & =w(\langle x\rangle,\langle y\rangle)+\ldots \\
\operatorname{Var}\left(w^{2}\right) & =\left\langle(w-\langle w\rangle)^{2}\right\rangle \\
& =\left(\frac{\partial w}{\partial x}\right)^{2} \operatorname{Var}\left(x^{2}\right)+\left(\frac{\partial w}{\partial y}\right)^{2} \operatorname{Var}\left(y^{2}\right)+2 \frac{\partial w}{\partial x} \frac{\partial w}{\partial y} \operatorname{Var}(x y), \\
\operatorname{Var}\left(\left(\frac{x}{y}\right)^{2}\right) & =\frac{1}{y^{2}} \operatorname{Var}\left(x^{2}\right)+\frac{x^{2}}{y^{4}} \operatorname{Var}\left(y^{2}\right)-\frac{2 x}{y^{3}} \operatorname{Var}(x y) .
\end{aligned}
$$

Thus, the standard deviation of the ratio is reduced by the correlation $\operatorname{Var}(x y)$ between the two contracts.

Figure 6 shows ratios of Basis-Point Volatilities (BPV) of front/back contracts with bands of their standard deviations.

\subsection{Standard Deviation of Differenced Volatilities}

To determine whether there is reason to look further into the existence of a volatility of the volatility, we test to see if there is any support for writing the volatility $\sigma$, the standard deviation of the differences of the volatilities (SDDBPV) as a simple stochastic process,

$$
\begin{aligned}
\frac{d \hat{\sigma}}{d t} & =\hat{\mu}+\epsilon \eta, \\
\hat{\mu} & =\langle d \hat{\sigma}\rangle=\langle\hat{\sigma}(t)-\hat{\sigma}(t-d t)\rangle, \\
\epsilon & =\left(\frac{n}{n-1}(\langle d \hat{\sigma}\rangle-\langle d \hat{\sigma}\rangle)^{2}\right)^{1 / 2},
\end{aligned}
$$

where $n$ is the number of data points (here 20) and $d t=1$.

It is reasonable to examine $\hat{\sigma}$ as the BPV, since we then are not assuming a specific BS model for a functional form of the noise, especially in a context where we are generalizing the BS model. However, it can be questioned, in the context of a BS model, where $\hat{\sigma}=\sigma y$ and $y$ is the yield, whether the volatility in $\hat{\sigma}$ arises primarily as an "artifact" from the volatility in the $y$ factor. Therefore, we also show that the volatility of $\sigma$, SDDBSV is not ncgligible, and when it is scaled by an average value of the yield, $\langle y\rangle$, we find that the standard deviation of the differences of the BS volatilities (SDDBSV) is given by 


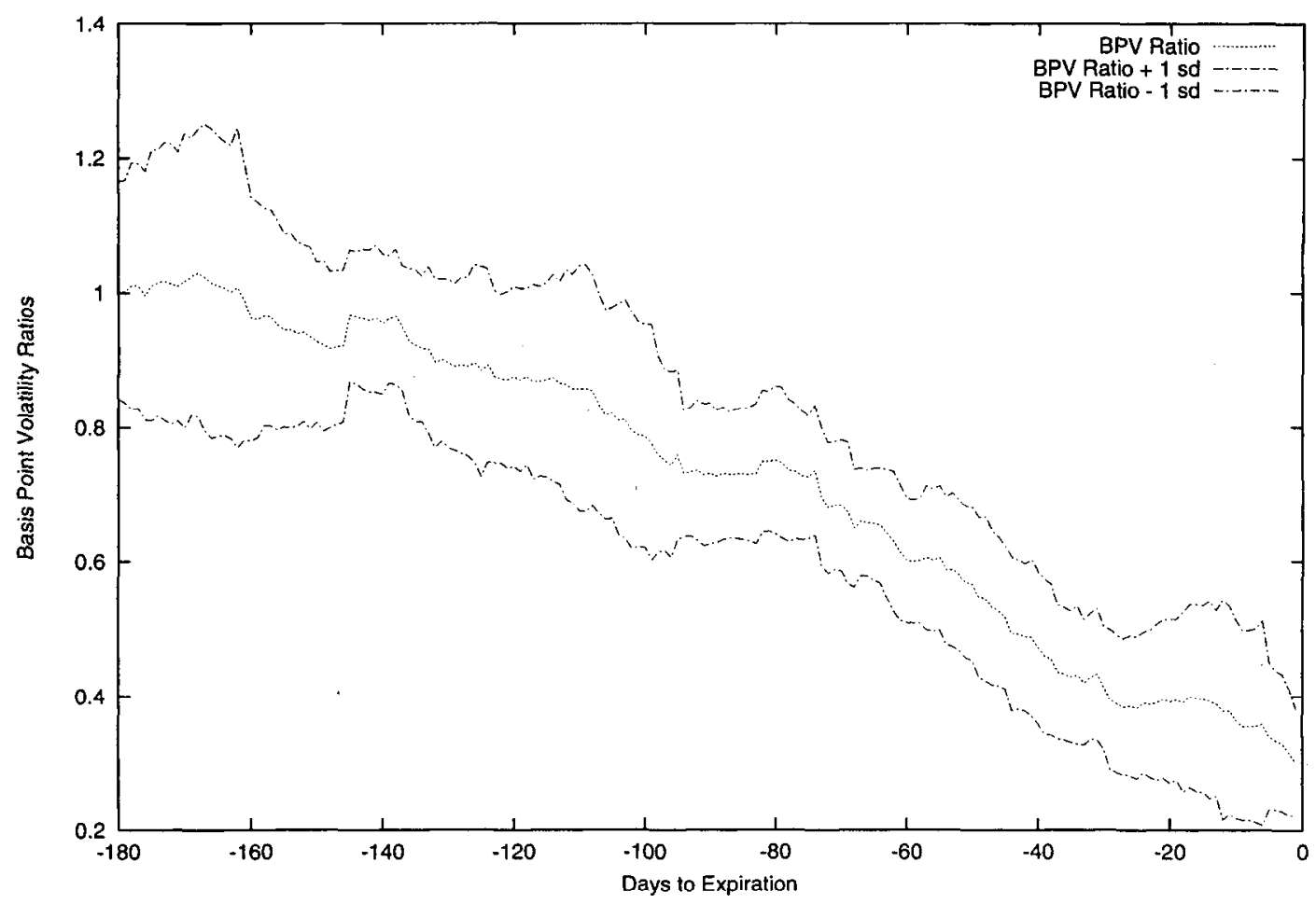

Figure 6. Ratios of Basis-Point Volatilities (BPV) of front/back contracts with bands of their standard deviations, marked to the number of days to expiration of the front contract.

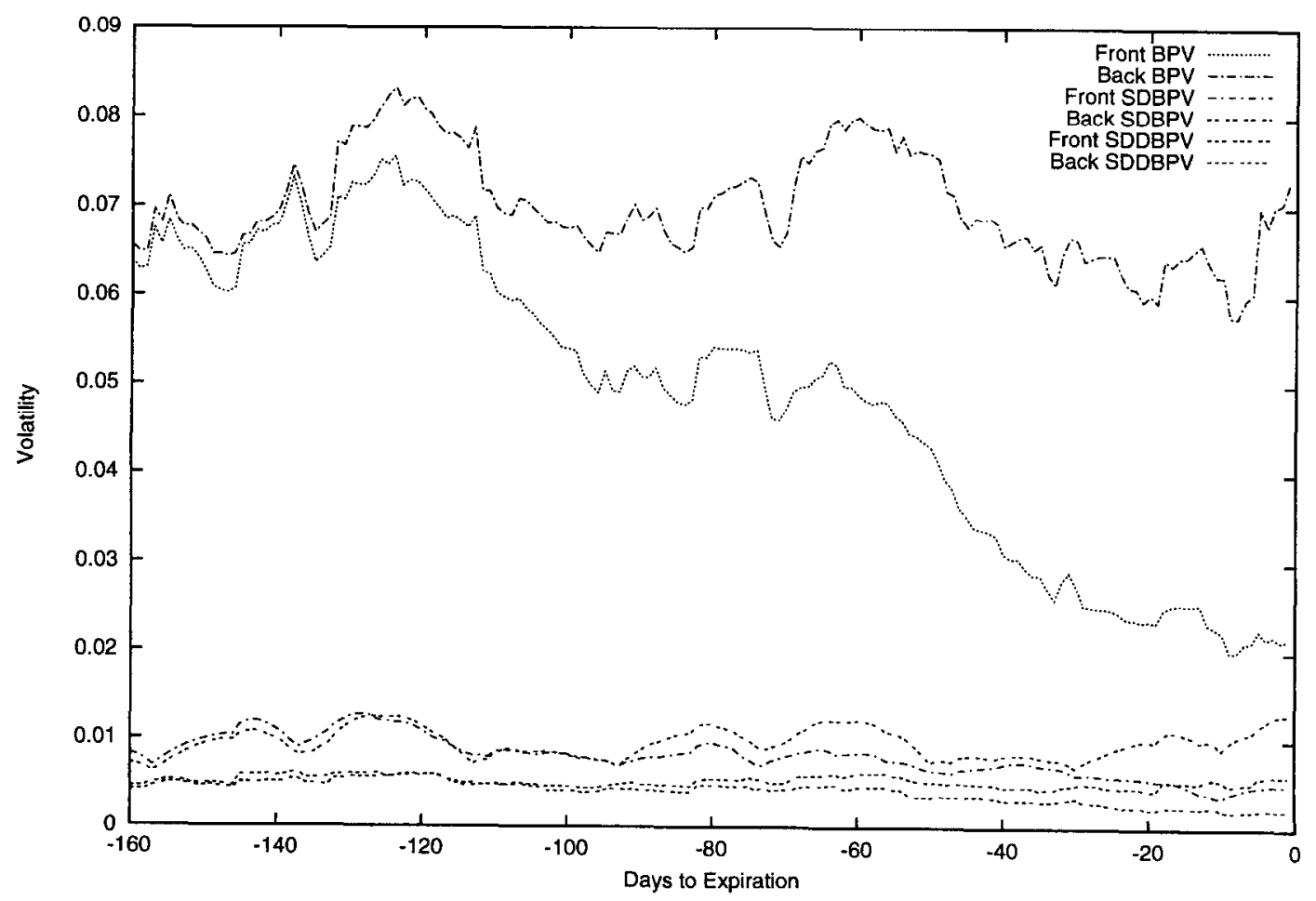

Figure 7. Comparison of Basis-Point Volatility (BPV), standard deviation of BPV (SDBPV), and standard deviation of differenced BPV (SDDBPV), for front and back contracts. 
Figure 7 gives a comparison of Basis-Point Volatility (BPV), standard deviation of BPV (SDBPV), and standard deviation of differenced BPV (SDDBPV), for front and back contracts. The SDBPV illustrate that there exists a distribution of volatilities about the mean volatility. The SDDBPV illustrate that this distribution likely is a stochastic process with a constant diffusion.

Figure 8 gives a comparison of standard deviation of differenced Basis-Point Volatility (SDDBPV) with and standard deviation of differenced Black-Scholes Volatility (SDDBSV), for front and back contracts. The SDDBSV have been scaled to the SDDBPV by multiplying them by the rounded average of the yields, i.e., 6.0. Note that after scaling, thcy consistently lie close to each other. Thus, both the BPV and BSV have volatilities that can be considered to be stochastic processes with constant diffusion.

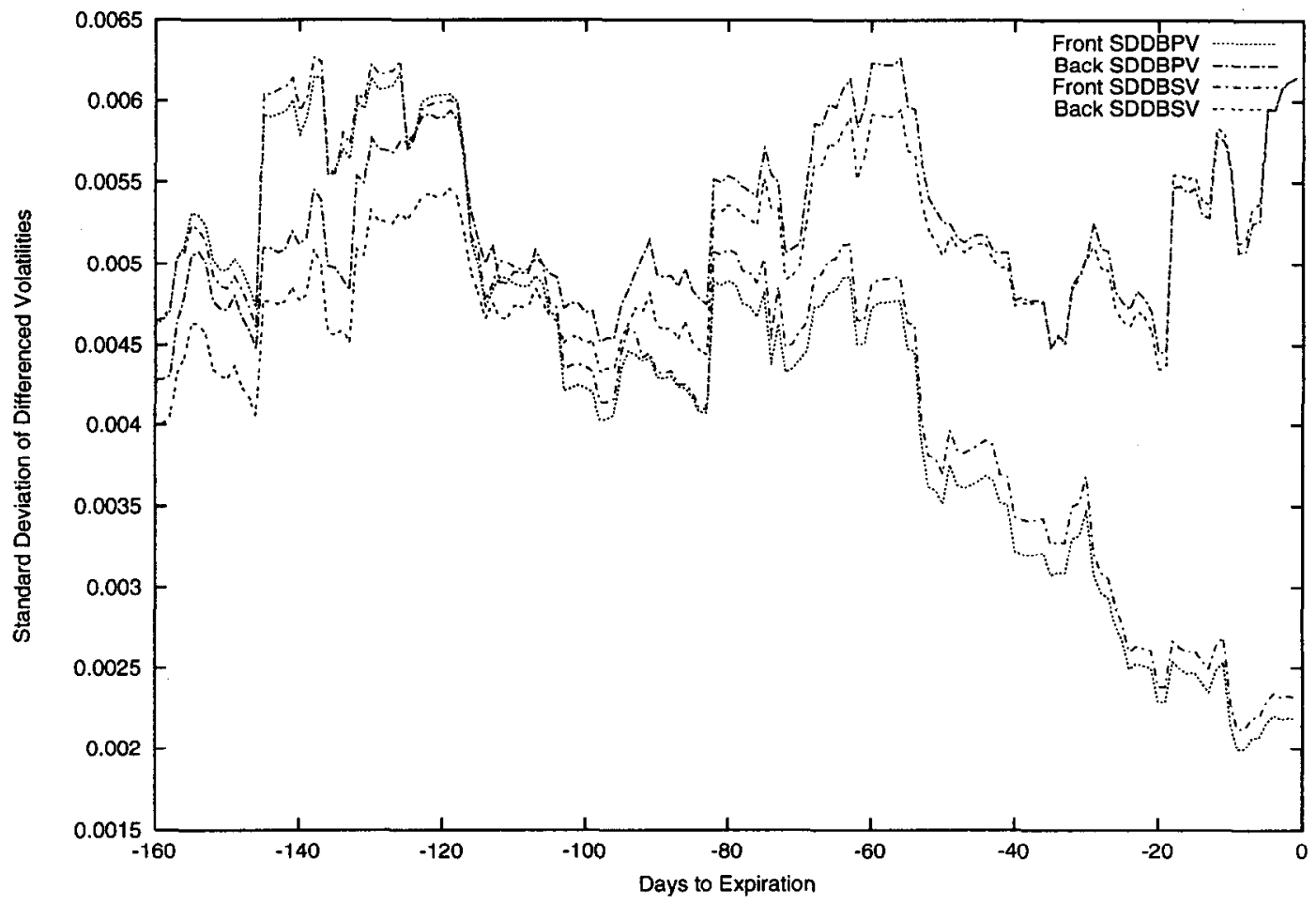

Figure 8. Comparison of standard deviation of differenced Basis-Point Volatility (SDDBPV) with and standard deviation of differenced Black-Scholes Volatility (SDDBSV), for front and back contracts. The SDDBSV have been scaled to the SDDBPV by multiplying them by the rounded average of the yields, i.e., 6.0.

\subsection{Implied Volatility}

The standard implied BS volatility is calculated as an average over the two nearest puts and calls to the at-the-money strike, of the volatilities derived from an inverse BS solution using the actual trading prices of the option. The use of the implied versus the historical volatility in trading varies from trader to trader, but here we wish to simply demonstrate that the implied volatility itself, similar to the historical volatility, has a distribution and likely is itself a stochastic process. For the implied volatility, the relation between the basis-point implied volatility (BPIV) and the BS implied volatility (BSIV) is simply

$$
\mathrm{BPIV}=y \mathrm{BSIV} \equiv y \sigma .
$$

The back month BSIV is taken from trading on the midcurve options, as discussed above. The data appears to be incomplete for various reasons, e.g., lack of trading, etc. 


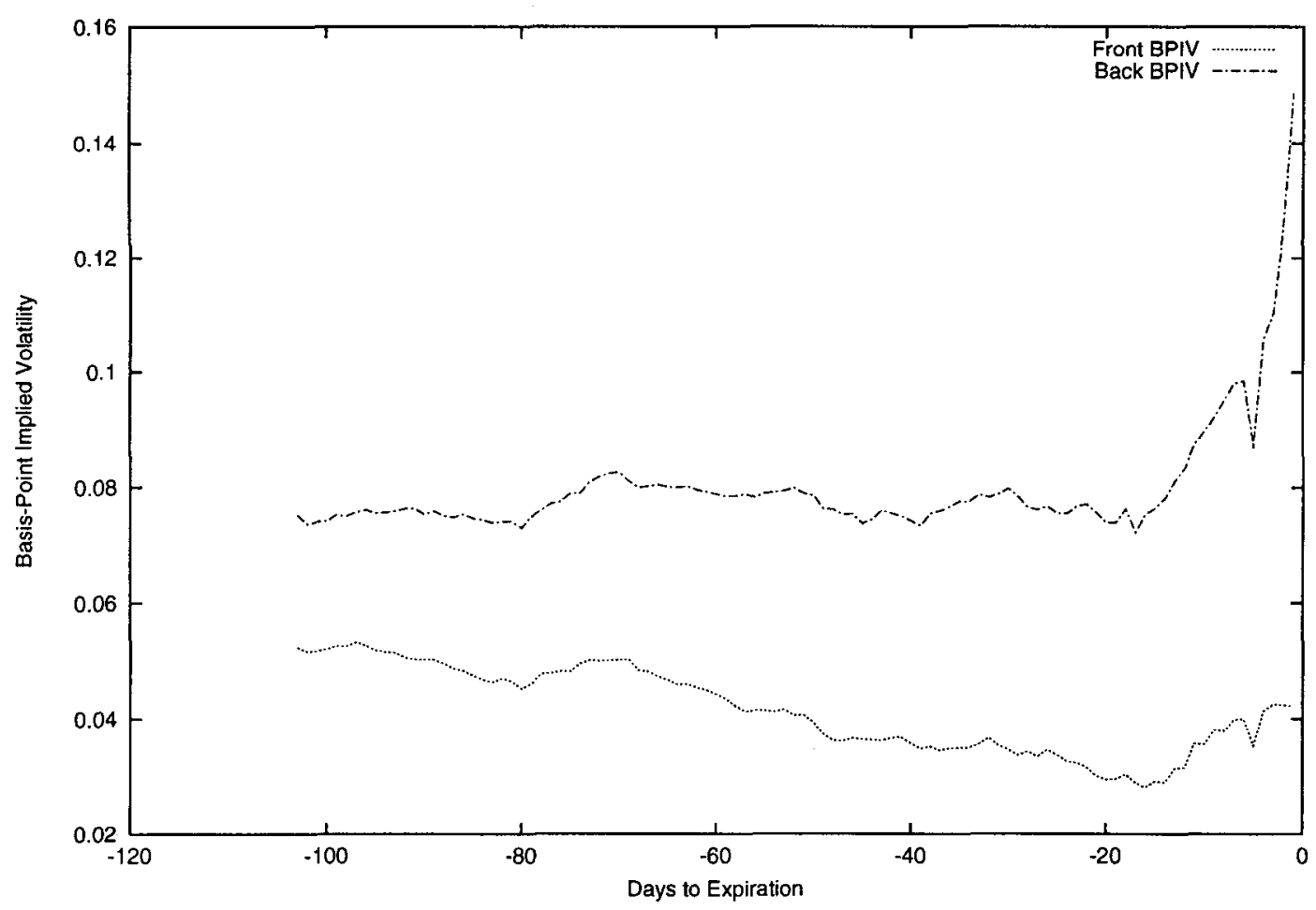

Figure 9. Basis-Point Implied Volatility (BPIV) of front and back contracts.

Figure 9 shows the Basis-Point Implied Volatility (BPIV) of front and back contracts. We have multiplied the differenced Black-Scholes Implied Volatility (BSIV) by a factor of 6 (a rough average of the yields) to scale these to other basis-point volatilities in this paper.

\subsubsection{Standard deviation of differenced implied volatility}

Figure 10 shows the standard deviation of differenced Basis-Point Implied Volatility (SDDBPIV) of front and back contracts. Note that, similar to the results above with respect to the historical volatilities, this illustrates that the volatility of the implied volatilities appears to be a stochastic process with constant diffusion.

\section{MULTIVARIATE NONLINEAR NONEQUILIBRIUM MARKETS}

It is clear that during very volatile markets, the BS formalism is both not as applicable nor is it as practically applicable (orders cannot get filled, markets close, etc.) as during relatively "normal" market conditions.

In such highly nonlinear nonequilibrium contexts, it may be useful to look for other criteria that can maximize profits and reduce risk. A formal approach to a statistical mechanics of nonlinear financial markets (SMFM) [4] has been shown to be useful in other disciplines, e.g., analyses of electroencephalography and combat scenarios, as documented in several papers available from the http://www. ingber. com archive. The development of powerful gencric numerical techniques, e.g., adaptive simulated annealing (ASA) optimization [17] and path-integral (PATHINT) calculations have been demonstrated to be give numerical support to the sophisticated SMFM algebra, in general to the several disciplines just mentioned, and specifically to financial markets [5-8]. This formalism may be useful as well for nonlinear nonequilibrium derivatives, e.g., by developing general "Euler-Lagrange" equations of motion and canonical momenta indicators (CMI) of multivariate markets. 


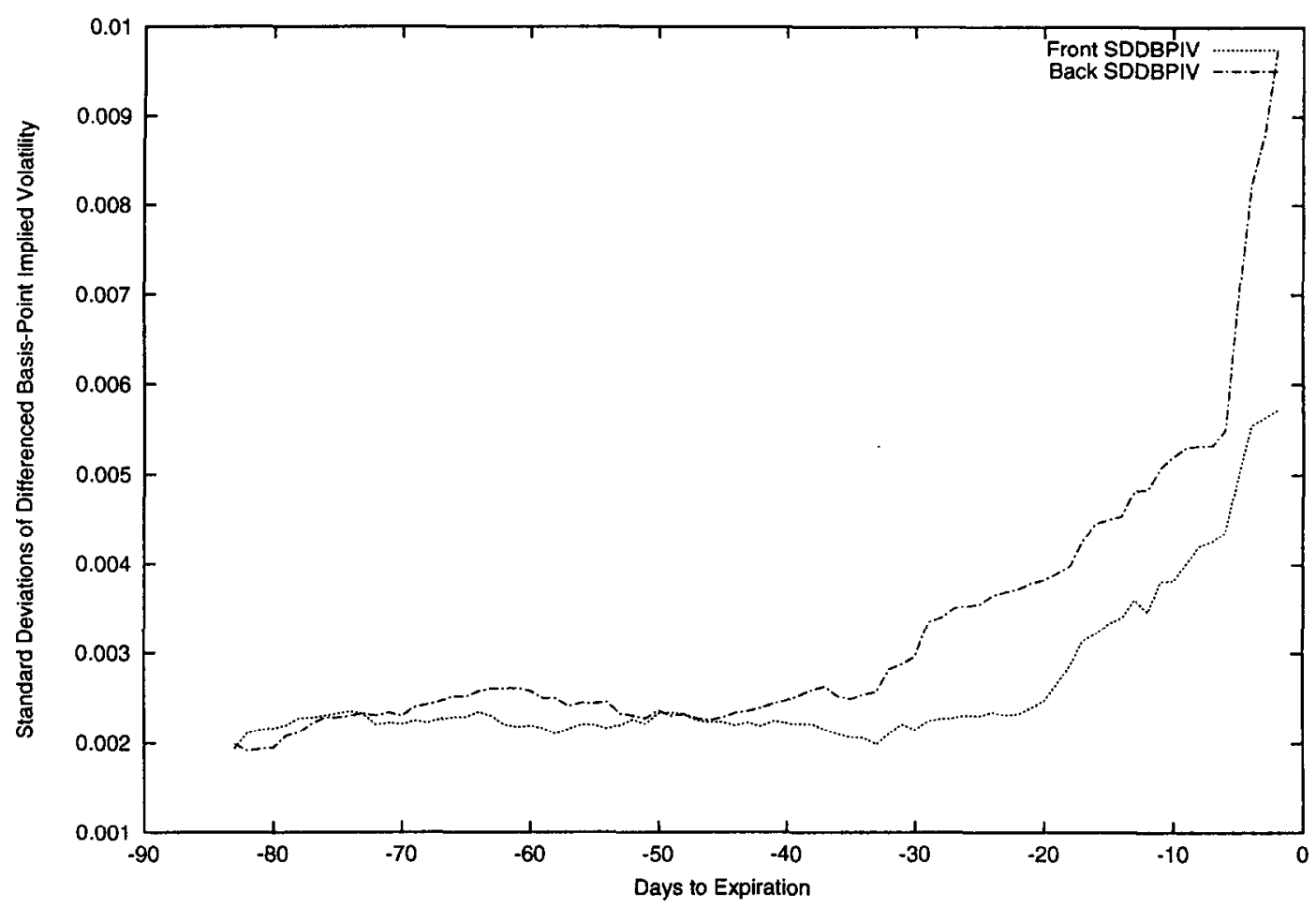

Figure 10. Standard deviation of differenced Basis-Point Implied Volatility (SDDBPIV) of front and back contracts.

\subsection{Generalized BS Mode}

In the context of the empirical evidence presented in the above section, a reasonable argument can be given for some degree of independent noise in an option addition to that derived from its underlying security. It is now widely accepted that volatility of traded securities is primarily incurred during actual trading days, e.g., 252 days/year, such that yearly rates of volatility are scaled accordingly $[18,19]$. Put another way, the activity of trading may induce volatility in any given security, based in large part on how traders perceive the market. It is not uncommon, for example, to find relatively high implied volatilities of options on relatively low volatilities of their underlying securities.

Thus, we consider adding an independent source of noise to the option, in addition to considering the primary source of noise from the underlying being a stochastic process.

For example, consider a plausible generalization of the basic stochastic equations above defining a stock and its option,

$$
\begin{aligned}
& \frac{d S}{S}=\sigma d X+\mu d t \\
& d S=\sigma S d X+\left(\mu-\frac{1}{2} \sigma^{2}\right) S d t \\
& d V=\nu d Y+\sigma S \frac{\partial V}{\partial S} d X+\left(\mu S \frac{\partial V}{\partial S}+\frac{1}{2} \sigma^{2} S^{2} \frac{\partial^{2} V}{\partial S^{2}}+\frac{\partial V}{\partial t}\right) d t \\
& d \sigma=\gamma d Z+\mu_{\sigma} d t
\end{aligned}
$$

where here $X, Y$ and $Z$ are independent random processes.

\subsection{Discretizations and Riemannian Geometry}

Coupling of variables in markets are included in a straight-forward manner in the above pathintegral formalism, and their parameters are readily optimized using ASA. It is convenient to use 
a more general compact notation for this purpose. For example, in terms of variables labeled by $G$, with drifts $f^{G}$ and metric $g_{G G^{\prime}}$ (the inverse of the covariance matrix $g^{G G^{\prime}}$ ),

$$
\begin{aligned}
M^{G} & =\left(\begin{array}{c}
S \\
V \\
\sigma
\end{array}\right), \\
f^{G} & =\left(\begin{array}{c}
\left(\mu-\frac{1}{2} \sigma^{2}\right) S \\
\beta \\
\mu_{\sigma}
\end{array}\right), \\
\beta & =\mu S \Delta+\Theta+\frac{1}{2}\left(\sigma S^{\prime}\right)^{2} \Gamma, \\
\hat{g}_{j}^{G} & =\left(\begin{array}{ccc}
\sigma S & 0 & 0 \\
\sigma S & \nu & 0 \\
0 & 0 & \gamma
\end{array}\right), \\
g^{G G^{\prime}} & =\hat{g}_{j}^{G} \hat{g}_{j}^{G^{\prime}} .
\end{aligned}
$$

In this notation, the Itô stochastic equations for $S$ and $V$ are

$$
\dot{M}^{G}=f^{G}+\hat{g}_{j}^{G} \eta^{j}
$$

where the Einstein summation convention is used, wherein factors with repeated indices are summed over. The Lagrangian in the prepoint discretized representation is given as

$$
\begin{aligned}
L & =\frac{1}{2}\left(\dot{M}^{G}-g^{G}\right) g_{G G^{\prime}}\left(\dot{M}^{G^{\prime}}-g^{G^{\prime}}\right)-\Phi, \\
g^{G} & =f^{G},
\end{aligned}
$$

where $\Phi$ is an additional "potential" term, here zero.

Note that if the above stochastic differential equations were written in the Stratonovich midpoint discretization, then we would have calculated

$$
\begin{aligned}
g^{G} & =f^{G}+\frac{1}{2} \hat{g}_{j}^{G^{\prime}} \hat{g}_{j}^{G}, G^{\prime} \\
& =\left(\begin{array}{c}
\mu S \\
\beta \\
\mu_{\sigma}
\end{array}\right), \\
{[\cdots]_{, G} } & =\frac{\partial[\cdots]}{\partial G} .
\end{aligned}
$$

The one-dimensional BS equation (1) can be written similarly, where $V$ is the short-time conditional "probability", only $M^{G}=S$ exists in $L$, and $\Phi=-r$.

There are of course other discretizations than the Itô prepoint discretization of stochastic differential equations such as equation (4), related to other differential-operator orderings of partial differential equations such as equation (1), also related to other transformations of variables in Lagrangians such as equation (24) [15]. This can be understood in the Stratonovich (midpoint discretized) representation for more than two variables for nonconstant diffusions, where it is seen that a Riemannian geometry is induced by the metric $g_{G G^{\prime}}$, exhibiting the invariance of the basic probability distribution under this geometry [4].

For example, the Lagrangian in in equation (24) is in the Itô prepoint representation. In the Stratonovich midpoint discretization, the path integral for the long-time evolution of the probability distribution in terms of multiple foldings of the short-time distributions is

$$
\begin{aligned}
P\left[M_{t} \mid M_{t_{0}}\right] d M(t) & =\int \cdots \int \underline{D} M \exp (-S) \delta\left[M\left(t_{0}\right)=M_{0}\right] \delta\left[M(t)=M_{t}\right] \\
S & =\min \int_{t_{0}}^{t} d t^{\prime} L
\end{aligned}
$$




$$
\begin{aligned}
\underline{D} M & =\lim _{u \rightarrow \infty} \prod_{r h o=1}^{u+1} g^{1 / 2} \prod_{G}(2 \pi \delta t)^{-1 / 2} d M_{\rho}^{G}, \\
L\left(\dot{M}^{G}, M^{G}, t\right) & =\frac{1}{2}\left(\dot{M}^{G}-h^{G}\right) g_{G G,}\left(\dot{M}^{G^{\prime}}-h^{G^{\prime}}\right)+\frac{1}{2} h_{; G}^{G}+R / 6-\Phi, \\
h^{G} & =g^{G}-\frac{1}{2} g^{-1 / 2}\left(g^{1 / 2} g^{G G^{\prime}}\right)_{, G^{\prime}}, \\
g_{G G^{\prime}} & =\left(g^{G G^{\prime}}\right)^{-1}, \\
g & =\operatorname{det}\left(g_{G G^{\prime}}\right), \\
h_{; G}^{G} & =h_{, G}^{G}+\Gamma_{G F}^{F} h^{G}=g^{-1 / 2}\left(g^{1 / 2} h^{G}\right)_{, G}, \\
\Gamma_{J K}^{F} & \equiv g^{L F}[J K, L]=g^{L F}\left(g_{J L, K}+g_{K L, J}-g_{J K, L}\right), \\
R & =g^{J L} R_{J L}=g^{J L} g^{J K} R_{F J K L}, \\
R_{F J K L} & =\frac{1}{2}\left(g_{F K, J L}-g_{J K, F L}-g_{F L, J K}+g_{J L, F K}\right)+g_{M N}\left(\Gamma_{F K}^{M} \Gamma_{J L}^{N}-\Gamma_{F L}^{M} \Gamma_{J K}^{N}\right) .
\end{aligned}
$$

(Some authors use $g=\operatorname{det}\left(g^{G G^{\prime}}\right)$, especially when it is convenient to identify $g$ with $\sigma^{2}$ in the limit of one variable.)

\subsection{Euler-Lagrange (EL) Equations}

The above three-variable set of stochastic differential equations for $S, V$, and $\sigma$ of course can be processed by the BS methodology, subject to linear algebraic constraints, essentially combining weighted sums of these equations to subtract out the noise terms in $d X$ and $d Y d X$ to contribute to a "risk-neutral"-type portfolio [10].

However, there exist other techniques to constrain such systems to paths that are not affected by "noise" to at least first- and second-order. For example, consider that the variational principle for $L$ possessed by this probability distribution $P, \delta L=0$, leads to Euler-Lagrange (EL) coupled second-order (in $t$ ) ordinary differential equations (given below for a more general multivariate system). These equations can possess "steady-state" solutions, which are "deterministic" in that they "ride" over the stochastic variables of the system.

In other words, the EL equations can lead to values of $\{\delta, \Gamma, \Theta$, vega, $\rho\}$ useful for "risk-neutral" hedging that are different from those obtained by the BS model. (Some economists would argue that additionally $\mu$ be set to $r$ to have these equations enforce risk-neutral hedging.) These new solutions do not have as restrictive underlying assumptions, and they may be useful especially in highly volatile markets.

\subsection{Most Probable Transitions}

Another set of coupled equations that can be useful if only most probable transition states are sought, are simple coupled first-order rate equations [20],

$$
\dot{M}^{G}=f^{G}-\frac{1}{2} g^{1 / 2}\left(g^{-1 / 2} g^{G G^{\prime}}\right)_{, G^{\prime}}
$$

\subsection{Canonical Momenta Indicators (CMI)}

The output of all this algebra need not be confined to complex algebraic forms or tables of numbers. Because $L$ possesses a variational principle, sets of contour graphs, at different longtime epochs of the path-integral of $P$ over its variables at all intermediate times, give a visually intuitive and accurate decision-aid to view the dynamic evolution of the scenario. 
For example, this Lagrangian approach permits a quantitative assessment of concepts usually only loosely defined,

$$
\begin{aligned}
\text { "Momentum" } & =\Pi^{G}=\frac{\partial L}{\partial\left(\frac{\partial M^{G}}{\partial t}\right)}, \\
\text { "Mass" } g_{G G^{\prime}} & =\frac{\partial^{2} L}{\partial\left(\frac{\partial M^{G}}{\partial t}\right) \partial\left(\frac{\partial M^{G}}{\partial t}\right)}, \\
\text { "Force" } & =\frac{\partial L}{\partial M^{G}}, \\
\text { "F } m \text { " : } \quad \delta L & =0=\frac{\partial L}{\partial M^{G}}-\frac{\partial}{\partial t} \frac{\partial L}{\partial\left(\frac{\partial M^{G}}{\partial t}\right)},
\end{aligned}
$$

where the last " $F=m a$ " equation is the set of EL equations discussed above. These physical entities provide another form of intuitive, but quantitatively precise, presentation of these analyses. For example, daily newspapers use this terminology to discuss the movement of security prices.

The CMI can be used to develop trading rules [8]. The extreme sensitivity of the CMI gives rapid feedback on the changes in trends as well as the volatility of markets. A time-locked moving average, yielding averages over each time in moving windows during which the model is fit to data, provides manageable indicators for trading signals.

\section{CONCLUSION}

We have presented empirical evidence that volatility of Eurodollar options possesses its own volatility, and that this volatility of volatility appears to be a stochastic process itself.

We have given a theoretical approach to incorporate this stochastic volatility process into a generalization of the standard Black-Scholes (BS) model of options.

We intend to further examine these empirical and theoretical issues in Eurodollar markets as well as in other financial markets.

\section{APPENDIX \\ UNBIASED ESTIMATOR FOR STANDARD DEVIATION}

Pick a sample of $x_{i}$, with a sample mean $\bar{x}$,

$$
\bar{x}=\frac{1}{n} \sum_{i} x_{i}
$$

and sample variance $s^{2}$,

$$
s^{2}=\frac{1}{n} \sum_{i}\left(x_{i}-\bar{x}\right)^{2} .
$$

The $x_{i}$ are assumed to be representative measures of a random variable $x$, with expected value, i.e., expectation, $\langle x\rangle$ taken with respect to an assumed probability distribution $f(x)$, where $f$ is defined by $\{$ mean, var $\}=\left\{\mu, \sigma^{2}\right\}$. The expectation of the sample is

$$
\langle\bar{x}\rangle=\frac{1}{n} \sum\left\langle x_{i}\right\rangle=n \frac{\mu}{n}=\mu .
$$


The variance of $\bar{x}$ is given by

$$
\begin{aligned}
\left\langle(\bar{x}-\langle\bar{x}\rangle)^{2}\right\rangle & =\left\langle\left(\frac{1}{n} \sum_{i} x_{i}-\mu\right)^{2}\right\rangle=\frac{1}{n^{2}}\left\langle\sum_{i} \sum_{j}\left(x_{i}-\mu\right)^{2}\right\rangle \\
& =\frac{1}{n^{2}}\left(\sum_{i} \sum_{j \neq i}\left\langle\left(x_{i}-\mu\right)\right\rangle\left\langle\left(x_{j}-\mu\right)\right\rangle+n \sum_{i}\left\langle\left(x_{i}-\mu\right)^{2}\right\rangle\right)=\frac{1}{n} \sigma^{2} .
\end{aligned}
$$

As an intermediate helpful step, consider

$$
\begin{aligned}
\left\langle\left(x_{i}-\bar{x}\right)^{2}\right\rangle & =\left\langle\left(\left(x_{i}-\mu\right)-(\bar{x}-\mu)\right)^{2}\right\rangle \\
& =\left\langle\left(x_{i}-\mu\right)^{2}\right\rangle+\left\langle(\bar{x}-\mu)^{2}\right\rangle-2\left\langle\left(x_{i}-\mu\right)(\bar{x}-\mu)\right\rangle \\
& =\sigma^{2}+\frac{1}{n} \sigma^{2}-2\left\langle\left(x_{i}-\mu\right)(\bar{x}-\mu)\right\rangle .
\end{aligned}
$$

The last term is further developed using

$$
\begin{aligned}
\left\langle\left(x_{i}-\mu\right)\left(\frac{1}{n} \sum_{j} x_{j}-\mu\right)\right\rangle & =\left\langle\left(x_{i}-\mu\right) \frac{1}{n} \sum_{j}\left(x_{j}-\mu\right)\right\rangle \\
& =\frac{1}{n}\left(\sigma^{2}+\sum_{j \neq i}\left\langle\left(x_{i}-\mu\right)\left(x_{j}-\mu\right)\right\rangle\right)=\frac{1}{n} \sigma^{2} .
\end{aligned}
$$

Summing over the result of this intermediate step,

$$
\left\langle s^{2}\right\rangle=\frac{1}{n} \sum_{i}\left\langle\left(x_{i}-\bar{x}\right)^{2}\right\rangle=\frac{1}{n} n\left(\sigma^{2}+\frac{1}{n} \sigma^{2}-\frac{2}{n} \sigma^{2}\right)=\frac{n-1}{n} \sigma^{2} .
$$

In summary,

$$
\mathrm{SD}-\sqrt{\frac{n}{n-1} s^{2}}=\sqrt{\frac{1}{n-1} \sum_{i}\left(x_{i}-\bar{x}\right)^{2}}
$$

is an unbiased estimator for the standard deviation, since its expectation, $\langle\mathrm{SD}\rangle=\sigma$, is independent of $n$.

In this paper, the standard deviation is simply written as

$$
\text { standard deviation }(x)=\left\langle\frac{n}{n-1}(x-\langle x\rangle)^{2}\right\rangle^{1 / 2} .
$$

\section{REFERENCES}

1. L. Ederington and W. Guan, Is implied volatility an informationally efficient and effective predictor of future volatility?, University of Oklahoma, Norman, OK, (1998).

2. G. Bakshi, C. Cao and Z. Chen, Pricing and hedging long-term options, Pennsylvania State University, University Park, PA, (1998).

3. L. Ingber, Some applications of statistical mechanics of financial markets, LIR-98-1-SASMFM, Lester Ingber Research, Chicago, IL, (1998).

4. L. Ingber, Statistical mechanics of nonlinear nonequilibrium financial markets, Math. Modelling 5 (6), 343$361,(1984)$.

5. L. Ingber, Statistical mechanical aids to calculating term structure models, Phys. Rev. A 42 (12), 7057-7064, (1990).

6. L. Ingber, M.F. Wehner, G.M. Jabbour and T.M. Barnhill, Application of statistical mechanics methodology to term-structure bond-pricing models, Mathl. Comput. Modelling 15 (11), 77-98, (1991).

7. L. Ingber, Statistical mechanics of nonlinear nonequilibrium financial markets: Applications to optimized trading, Mathl. Comput. Modelling 23 (7), 101-121, (1996). 
8. L. Ingber, Canonical momenta indicators of financial markets and neocortical EEG, In Progress in Neural Information Processing, (Edited by S.-I. Amari, L. Xu, I. King and K.-S. Leung), pp. 777-784, Springer, New York, (1996).

9. Federal Rescrve Bank, Instruments of the Money Markets, Seventh Edition, Federal Reserve Bank of Richmond, Richmond, VA, (1993).

10. J.C. Hull, Options, Futures, and Other Derivatives, Third Edition, Prentice Hall, Upper Saddle River, NJ, (1997).

11. P. Wilmott, S. Howison and J. Dewynne, The Mathematics of Financial Derivatives, Cambridge University Press, Cambridge, (1995).

12. M.F. Wehner and W.G. Wolfer, Numerical evaluation of path-integral solutions to Fokker-Planck equations. I., Phys. Rev. A 27, 2663-2670, (1983).

13. L. Ingber, H. Fujio and M.F. Wehner, Mathematical comparison of combat computer models to exercise data, Mathl. Comput. Modelling 15 (1), 65-90, (1991).

14. L. Ingber, Statistical mechanics of neocortical interactions: Path-integral evolution of short-term memory, Phys. Rev. 49 (5B), 4652-4664, (1994).

15. F. Langouche, D. Roekaerts and E. Tirapegui, Punctional Integration and Semiclassical Expansions, Reidel, Dordrecht, The Netherlands, (1982).

16. G. Walther, Absence of correlation between the solar neutrino flux and the sunspot number, Phys. Rev. Lett. 79 (23), 4522-1521, (1998).

17. L. Ingber, Adaptive Simulated Annealing (ASA), Global optimization C-code, Lester Ingber Research, Chicago, IL, (1993).

18. E.E. Fama, The behavior of stock market prices, J. Business 38, 31-105, (1965).

19. K.R. French, Stock returns and the weekend effect, J. Finan. Econ. 8, 55-69, (1980).

20. H. Dekker, On the most probable transition path of a general diffusion process, Phys. Lett. A 80, 99-101, (1980). 